\section{Preventive Effect of Somatostatin on Pancreatitis and Hyperamylasemia after Therapeutic ERCP}

Sir,

Endoscopic retrograde cholangiopancreatography (ERCP) is one of the important diagnostic and treatment methods for hepatobiliary and pancreatic diseases. ${ }^{1}$ Therapeutic ERCP has many complications for its many operations, long operating time, and many applications. Pancreatitis and hyperamylasemia are common complications after ERCP. Despite the improvements in ERCP technology in recent years and the growing experience of endoscopic surgeons, the incidence of pancreatitis after ERCP has not decreased, while the risk is still high in selected patients. ${ }^{2}$ At present, pancreatic duct stents and drug prevention methods are mostly used in clinical practice, but the cost of pancreatic duct stent is expensive and there are some limitations, so that drug prevention is the focus of clinical attention. Somatostatin is a 14-aminoacid containing peptide that is synthesized in gastric mucosal cells and pancreatic cells. 3 In recent years, the authors have observed that somatostatin has a better preventive effect on pancreatitis and hyperamylasemia after therapeutic ERCP.

A total of 150 patients with therapeutic ERCP were selected as subjects, with age more than 18 years, of both genders and the serum amylase was normal before operation. There was no contraindication of ERCP. The patients were randomly divided into somatostatin group and control group, with 75 cases in each group. All patients were given intravenous drip of saline $500 \mathrm{~mL}$ before ERCP. The control group received antiinflammatory, fluid replacement, and symptomatic supportive treatment after ERCP. In somatostatin group, $3 \mathrm{mg}$ of somatostatin was administered 30 minutes before surgery and continued for 12 hours; while antiinflammatory, fluid replacement and symptomatic supportive treatment were given after ERCP. The total incidence of postoperative pancreatitis was $19.33 \%$ (29 cases) and of hyperamylasemia was $5.33 \%$ (8 cases) in all patients. Among them, the incidence of postoperative pancreatitis $(9.33 \%, 7$ cases) and hyperamylasemia $(1.33 \%, 1$ case) in somatostatin group was significantly lower than that in control group (29.33\%, 22 cases) and $(9.33 \%, 7$ cases, $p=0.002,0.029$, respectively).
Application of somatostatin before ERCP could effectively prevent postoperative pancreatitis and hyperamylasemia. Previous studies have also concluded that the administration of somatostatin is useful in preventing pancreatitis in patients undergoing therapeutic ERCP. 4 Its mechanism may be that somatostatin inhibits the exocrine function of the pancreas, which could reduce the secretion of digestive juice. It may indirectly affect the exocrine function of the pancreas by inhibiting secretin and cholecystokinin. In addition, somatostatin could also down-regulate the cascade effect of cytokines, protecting the pancreas and preventing the pancreatitis and hyperamylasemia after ERCP. 5 Although hyperamylasemia without clinical symptoms does not require special treatment, and most acute pancreatitis is also mild, but there is a risk of development of necrotizing pancreatitis that can endanger the life of the patients. Therefore, it is imperative that preventive storategies should be employed to reduce the incidence of these complications following therapeutic ERCP.

\section{REFERENCES}

1. Dilshad A, Salim A, Haq MIU, Amin J, Malik K, Alam A. Outcome of endoscopic management of post living donor liver transplant anastomotic strictures. J Coll Physicians Surg Pak 2018; 28:514-7.

2. Jeon J, Lim SU, Park $\mathrm{CH}$, Jun $\mathrm{CH}$, Park SY, Rew JS. Restoration of common bile duct diameter within 2 weeks after endoscopic stone retraction is a preventive factor for stone recurrence. Hepatobiliary Pancreat Dis Int 2018; 17:251-6.

3. Malaisse WJ. Paracrine control of glucagon release by somatostatin (Review). Int J Mol Med 2013; 33:491-8.

4. Lee KT, Lee DH, Yoo BM. The prophylactic effect of somatostatin on post-therapeutic endoscopic retrograde cholangiopancreatography pancreatitis: A randomized, multicenter controlled trial. Pancreas 2008; 37:445-8.

5. Andriulli A, Leandro G, Federici T, Ippolito A, Forlano R, lacobellis $A$, et al. Prophylactic administration of somatostatin or gabexate does not prevent pancreatitis after ERCP: an updated meta-analysis. Gastrointest Endosc 2007; 65:624-32.

Shouhe Zhao and Junhua Yu

Department of General Surgery, Pingshan District People's Hospital of Shenzhen, Shenzhen, Guangdong, 518118, China

Correspondence: Junhua Yu, Department of General Surgery, Pingshan District People's Hospital of Shenzhen, Shenzhen, Guangdong, 518118, China

E-mail: goqwrpu@163.com

Received: October 12, 2018; Accepted: November 04, 2018 